\title{
Herpetofauna of the Argentinean Impenetrable Great Chaco
}

\author{
Federico Pablo Kacoliris ${ }^{1}$, Igor Berkunsky ${ }^{2}$, Jorge Williams ${ }^{1}$ \\ 1 Sección Herpetología, Departamento de Vertebrados, Museo de La Plata. Universidad Nacional de La Plata. Paseo del \\ Bosque s/n, B1900FWA, La Plata, Argentina. E-mail: fedekacoliris@yahoo.com.ar. \\ 2 Departamento de Ecología, Genética y Evolución, Facultad de Ciencias Exactas y Naturales. Universidad de Buenos \\ Aires. Pabellón II, Ciudad Universitaria, C1428EHA, Buenos Aires, Argentina
}

Keywords: Amphibians, geographical distribution, herpetofauna, reptiles, conservation, Chaco, Argentina.

The Great Chaco is a region of nearly $1,000,000 \mathrm{~km}^{2}$ extended through the plains of Northern and central Argentina, Western Paraguay, Southeastern Bolivia, and the extreme Western edge of Mato Grosso do Sul state in Brazil (Pennington et al. 2000). Originally dominated by xerophytic semi-deciduous forests, the area has been extensively cleared for timber production and agriculture over the last century; only small patches of native vegetation remain (Zak and Cabido 2002).

The Impenetrable region (Northern region of the Chaco and Santiago del Estero Provinces and Eastern part of Salta Province, Argentina) is one of the biggest remnant patches of native dry forest (Bertonatti and Corcuera 2000). Most of the Impenetrable woodlands are found in the Chaco Province, and are legally protected by two fairly new Provincial Parks (P. P.): The "Fuerte Esperanza" P. P. (283 km²), created in 2001 (SGPC 2001), and the "Loro Hablador" P. P. $\left(175 \mathrm{~km}^{2}\right)$, created in 2004 (SGPC 2004).

Received 10 July 2006.

Accepted 5 December 2006.

Distributed December 2006.
Most available data on the herpetofauna of this area come from review works (Gallardo 1979, Cei 1980, 1993, Cabrera 1995, Leynaud and Bucher 1999, Richard 1999, Alvarez et al. 2002, Chebez et al. 2005). The region has been poorly explored and nearly all field studies were conducted on a few localities (Lavilla and Scrocchi 1991, Lavilla and Rougés 1992, Lavilla et al. 1995a,b, Perotti 1997, Leynaud and Bucher 2005). Herein, we present a list of amphibians and reptiles of the Impenetrable Chaco region. This work records new localities for most species and assesses if the Loro Hablador Provincial Parks are protecting a representative portion of herpetofauna richness.

The area surveyed is a flood plain characterized by a continuous dry forest dominated by "quebrachos" (Aspidosperma quebracho-blanco, Schinopsis lorentzii) and by the absence of permanent wetlands, rivers, and streams (Cabrera 1976). The average annual rainfall is about $600 \mathrm{~mm}$; $75 \%$ occurs between November and March (for a thorough vegetation characterization, see Morello and Adámoli 1974). We surveyed the area during the spring and summer months between 1999 and 2006 (total 22 months of fieldwork). We visited eight 
localities from Chaco Province: P. P. Loro

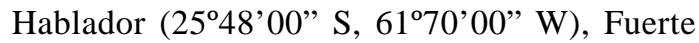

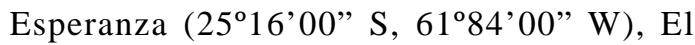
Torito $\left(25^{\circ} 22^{\prime} 00^{\prime}\right.$ S, 62 $02^{\circ} 00^{\prime}$ ' W), Luján $\left(25^{\circ} 20^{\prime} 00^{\prime}\right.$ S, 62 $\left.2^{\circ} 30^{\prime} 00^{\prime \prime} \mathrm{W}\right)$, Chiva Mora

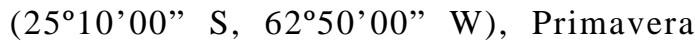
(2505'00” S, 62 $50^{\circ} 00^{\prime}$ ' W), Rio Muerto (2510’00” S, 62 65'00” W), and Taco Pozo (2561'00” S, 6328'00” W) (Figure 1). Most of the work effort was concentrated at P. P. Loro Hablador.

Specimens were identified using available keys; most individuals were identified during the fieldwork (Cei 1980, 1993, Giraudo 2001). Examined material (pictures and specimens) are deposited at Museo de La Plata (MLP), Buenos Aires, Argentina and listed in Appendix I. We constructed a list of cited species for the Impenetrable following Lavilla and Scrocchi (1991), Lavilla and Rougés (1992), Cabrera (1995), Lavilla et al. (1995a,b), Perotti (1997), Leynaud and Bucher (1999, 2005), Alvarez et al. (2002), Nenda and Sccrochi (2004), Chebez et al. (2005), and Kretzschmar (2006).

We found 18 (64\%) of the 28 previously reported amphibian species. We also found one species (Pseudis paradoxa occidentalis) not cited before for this area (Table 1). We found 37 species $(82 \%)$ of the 45 cited reptilian species (Table 2).

Some records deserve special comments because of their zoogeographic and/or conservation implications. We found one "endangered" species (Chelonoidis chilensis); one "threatened" species (Boa constrictor occidentalis) two "vulnerable" species (Epicrates cenchria alvarezi and Polychrus acutirostris) and two "insufficiently known" species (Elachistochleis aff. bicolor and Stenocercus doellojuradoi) (SADS 2005).

The Chaco Tortoise (C. chilensis, Figure 2A) is a small terrestrial tortoise characteristic of the Impenetrable woodlands. Although it is an endangered species, the pet trade seems to be the current major treat (Lavilla et al .2001). Adults showed a high activity during the month

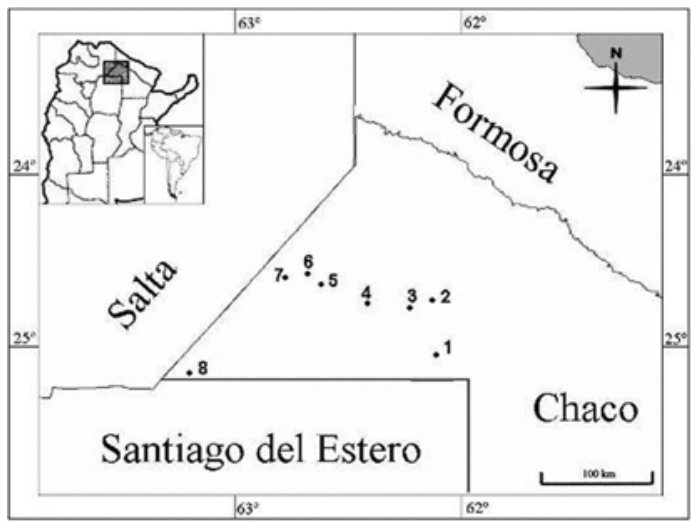

Figure 1 - Region of Impenetrable showing localities explored at this study: P. P. Loro Hablador (1), Fuerte Esperanza (2), El Torito (3), Luján (4), Chiva Mora (5), Primavera (6), Rio Muerto (7), and Taco Pozo (8).

of November and juveniles were only found in January.

The Red Tegu (Tupinambis rufescens) is under Appendix II of CITES. Local population of the Dry Chaco Region hunt this lizard as a source of food and to sale its skin to the international trade. Currently, there is a "Red Tegu National Harvesting Program" at the Impenetrable, whose goal is to achieve a sustainable management of the species (Fitzgerald 1994).

Stenocercus doellojuradoi is considered an “insufficiently known” species (Lavilla et al. 2000) due to the absence of data about their ecology, population densities and trade. We record this lizard all years in the P. P. Loro Hablador. This Park seems to be a good place to increase the knowledge about the biology of this species.

Two boid species coexist in Impenetrable Chaco area. The Argentine Constrictor Boa (B. constrictor occidentalis) is characteristic of dry woodlands in Argentina and Paraguay. Historically this species was captured for its skin and consequently populations have decreased in some areas (Lavilla et al. 2000). B. 
Table 1 - Amphibian species from the Impenetrable region showing localities explored in this study: P. P. Loro Hablador (1), Fuerte Esperanza (2), El Torito (3), Luján (4), Chiva Mora (5), Primavera (6), Rio Muerto (7), and Taco Pozo (8).

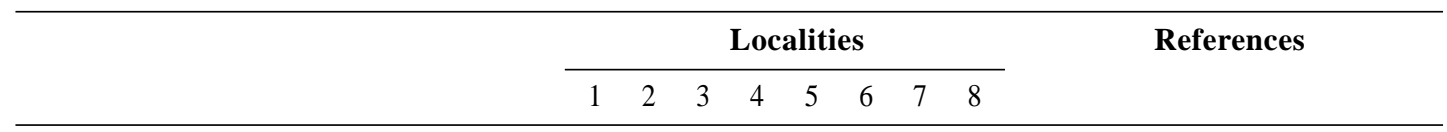

\section{BUFONIDAE}

Chaunus fernandezae

Chaunus granulosus major

Chaunus schneideri

CERATOPHRYIDAE

Ceratophrys cranwelli

Lepidobatrachus asper

Lepidobatrachus laevis

Lepidobatrachus llanensis

CYCLORAMPHIDAE

Odontophrynus americanus

Odontophrynus lavillai

HYLIDAE

Phyllomedusa hypochondrialis azurea

Phyllomedusa sauvagii

Pseudis paradoxus occidentalis

Pseudis minutus

Scinax acuminatus

Scinax nasicus

Trachycephalus venulosus

LEIUPERIDAE

Physalaemus albonotatus

Physalaemus biligonigerus

Physalaemus cuqui

Pleurodema tucumanum

\section{LEPTODACTYLIDAE}

Leptodactylus bufonius

Leptodactylus chaquensis

Leptodactylus elenae

Leptodactylus fuscus

Leptodactylus laticeps

Leptodactylus latinasus

Leptodactylus mystacinus

MICROHYLIDAE

Dermatonotus muelleri

Elachistocleis aff. bicolor

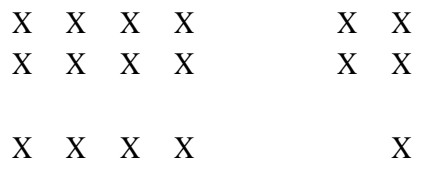

Alvarez et al. 2002

Alvarez et al. 2002

Lavilla et al. 1995a,

Perotti 1997, Alvarez et al. 2002 $\mathrm{X}$

Alvarez et al. 2002

X

$\mathrm{X}$

$\begin{array}{llllll}X & X & X & X & X & X \\ X & X\end{array}$

Alvarez et al. 2002

Alvarez et al. 2002

$\begin{array}{lll}X & X\end{array}$

X

X $\mathrm{X} \quad \mathrm{X}$

Alvarez et al. 2002

$\begin{array}{llll}X & X & X & X\end{array}$

$\mathrm{X}$

$\begin{array}{llll}X & X & X & X\end{array}$

$\mathrm{X}$

Lavilla and Rougés 1992,

Perotti 1997, Alvarez et al. 2002

$\begin{array}{llll}X & X & X & X\end{array}$

X X

$\mathrm{X}$

$\begin{array}{lllll}X & X & X & X & X\end{array}$

Alvarez et al. 2002

Lavilla et al. 1995a,

Alvarez et al. 2002 
Table 2 - Reptilian species of the Impenetrable region showing localities explored in this study: P. P. Loro Hablador (1), Fuerte Esperanza (2), El Torito (3), Luján (4), Chiva Mora (5), Primavera (6), Rio Muerto (7), and Taco Pozo (8).

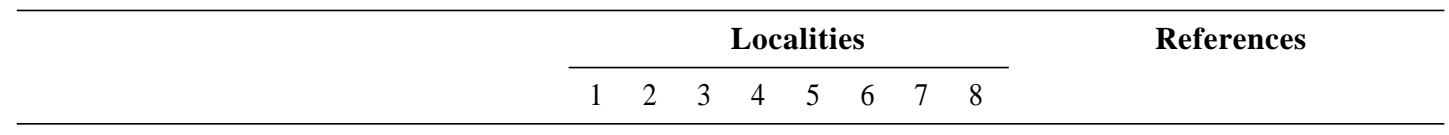

\section{CHELIDAE}

Acanthochelys pallidipectoris

CHELONIDAE

Chelonoidis chilensis

KINOSTERNIDAE

Kinosternon scorpioides

AMPHISBAENIDAE

Amphisbaena bolivica

Amphisbaena darwini

Anops kingi

Leposternon microcephalum

GEKKONIDAE

Homonota fasciata

Phyllopezus pollicaris przewalskyi

GYMNOPHTHALMIDAE

Cercosaura schreibersii

Vanzosaura rubricauda

LIOLAEMIDAE

Liolaemus chacoensis

POLYCHROTIDAE

Polychrus acutirostris

Urostrophus gallardoi

\section{TEIIDAE}

Ameiva ameiva

Cnemidophorus ocellifer

Teius teyou

Tupinambis rufescens

TROPIDURIDAE

Stenocercus doellojuradoi

Tropidurus etheridgei

Tropidurus spinulosus

SCINCIDAE

Mabuya frenata

BOIDAE

Boa constrictor occidentalis

Epicrates cenchria alvarezi
Lavilla et al. 1995a

X X X X

Lavilla et al. 1995a

X X

Lavilla et al 1995b, Alvarez et al. 2002, Leynaud and Bucher 2005

Alvarez et al. 2002

X

X X

Alvarez et al. 2002

X

X

$\mathrm{X}$

Lavilla et al. 1995b, Alvarez et al. 2002, Leynaud and Bucher 2005

$$
\begin{array}{lllllllll}
X & X & & & & & & \\
X & & & & & & & \\
X & X & X & X & X & X & X & X \\
X & X & X & & X & X & X & X
\end{array}
$$

$\mathrm{X}$

$\mathrm{X}$

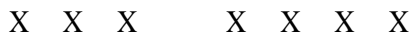

X X

$\begin{array}{llll}X & X & X & X\end{array}$

$\mathrm{X} \quad \mathrm{X}$ 
Table 2 - Continued.

\begin{tabular}{|c|c|c|c|c|c|c|c|c|c|}
\hline & \multicolumn{8}{|c|}{ Localities } & \multirow[t]{2}{*}{ References } \\
\hline & 1 & 2 & 3 & 4 & 5 & 6 & 7 & 8 & \\
\hline \multicolumn{10}{|l|}{ COLUBRIDAE } \\
\hline Boiruna maculata & $\mathrm{X}$ & $\mathrm{X}$ & & & & & & $\mathrm{X}$ & \\
\hline Chironius quadricarinatus maculoventris & & & & & & & $\mathrm{X}$ & & \\
\hline Leptodeira annulata pulchriceps & $\mathrm{X}$ & & & & & & & & \\
\hline Liophis guentheri & & & & & & & & & $\begin{array}{l}\text { Lavilla et al 1995b, Alvarez et al. } \\
\text { 2002, Chebez et al. } 2005\end{array}$ \\
\hline Liophis poecilogyrus caesius & $\mathrm{X}$ & & & & & & & & \\
\hline Liophis sagittifer modestus & $\mathrm{X}$ & & & & & & & & \\
\hline Lystrophis pulcher & $\mathrm{X}$ & $\mathrm{X}$ & $\mathrm{X}$ & & $\mathrm{X}$ & & & & \\
\hline Oxyrhopus rhombifer & $\mathrm{X}$ & & & & & & & & \\
\hline Phalotris punctatus & & & & & & & & & $\begin{array}{c}\text { Alvarez et al. 2002, Leynaud } \\
\text { and Bucher } 2005\end{array}$ \\
\hline Philodryas baroni & $\mathrm{X}$ & & & & & & $\mathrm{X}$ & & \\
\hline Philodryas mattogrossensis & $\mathrm{X}$ & & & & & $\mathrm{X}$ & & & \\
\hline Philodryas olfersii latirostris & $\mathrm{X}$ & & & & & & $\mathrm{X}$ & & \\
\hline Philodryas psammophidea & $\mathrm{X}$ & & & & & & & & \\
\hline Phimophis vittatus & $\mathrm{X}$ & & & & & & & & \\
\hline Sibynomorphus lavillai & $\mathrm{X}$ & & & & & & & & \\
\hline Waglerophis merremii & $\mathrm{X}$ & & & & & & $\mathrm{X}$ & & \\
\hline \multicolumn{10}{|l|}{ ELAPIDAE } \\
\hline Micrurus pyrrhocryptus & $\mathrm{X}$ & $\mathrm{X}$ & & & & & $\mathrm{X}$ & & \\
\hline \multicolumn{10}{|l|}{ LEPTOTYPHLOPIDAE } \\
\hline Leptotyphlops albipunctus & $\mathrm{X}$ & & & & & & & & \\
\hline Leptotyphlops unguirostris & $\mathrm{X}$ & & & & & & & & \\
\hline Typhlops brongersmianus & & & & & & & & & Alvarez et al. 2002 \\
\hline \multicolumn{10}{|l|}{ VIPERIDAE } \\
\hline Bothrops neuwiedii diporus & $\mathrm{X}$ & $\mathrm{X}$ & $\mathrm{X}$ & & & & $\mathrm{X}$ & & \\
\hline Crotalus durissus terrificus & $\mathrm{X}$ & & & & & & & & \\
\hline
\end{tabular}

constrictor occidentalis in particular is considered a threatened and protected species (Appendix I of CITES). The Argentine Rainbow Boa (E. cenchria alvarezi) occurs in the Northwestern region of Argentina, including Chaco and Yungas woodlands. Historically this species was also hunted for the skin trade and, as other boids, it is listed in Appendix II of CITES; however, we did not find evidence of current hunting by local population. We found
B. constrictor occidentalis both on the ground and on trees, whereas E. cenchria alvarezi was only observed inside tree cavities. Both species seem to be very common at the Impenetrable Chaco Region.

The Llanos Frog (Lepidobatrachus llanensis) is uncommon throughout its range and the populations are decreasing (IUCN 2006). The species range includes dry scrubland and semi-arid areas in Northern Argentina and 

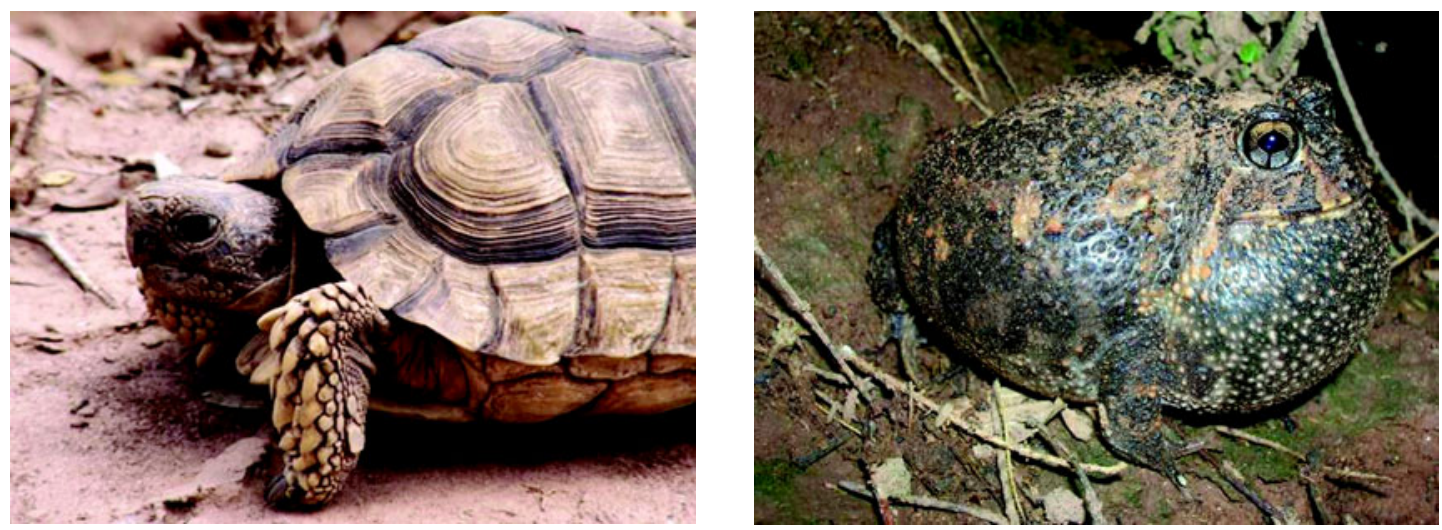

Figure 2 - (A) The Chaco Tortoise (Chelonoidis chilensis); (B) the Cei’s Escuerzo (Odontophrynus lavillai).

Western Paraguay. During the breeding season it appears to be a common species in ponds; we found adults in amplexus, tadpoles, and juveniles.

Cei's Escuerzo (Odontophrynus lavillai, Figura 2B) is endemic to Argentina, known only from the Provinces of Santiago del Estero, Salta, Chaco, and Jujuy. We recorded two new localities for this fossorial species. In both places, the species was found in artificial ponds.

This is the first herpetofaunal survey of the P. P. "Loro Hablador” (Chaco Province). The 50 species here recorded from this park represent respectively 52 and $77 \%$ of amphibian and reptilian species known from the Impenetrable region, therefore we think that this Park is protecting a significant number of total herpetofaunal species of the Impenetrable, including populations of vulnerable and threatened reptilian species. The knowledge of species distribution is crucial in understanding their natural history and in giving direction to future research and conservation efforts.

\section{Acknowledgements}

We thank B. Charpin, J. Carreras, Ch. de la Fourniére, A. Giraudo, L. Pagano, H. Povedano, J. Rojas, R. Rojas, and R. Ruggera, by their data contribution, determination of species and help in field work, and D. Ferraro and J. I. Areta by comments and suggestions. Gustavo Scrocchi and one anonymous reviewer provided useful comments on earlier drafts.

\section{References}

Alvarez, B., R. Aguirre, J. Céspedes, A. Hernando, and M. E. Tedesco. 2002. Atlas de Anfibios y Reptiles de las provincias de Corrientes, Chaco y Formosa (Argentina). I. Anuros, Cecílidos, Saurios, Anfisbénidos y Serpientes. Corrientes. Editorial Universitaria de la Universidad Nacional del Nordeste (EUDENE). 156 pp.

Bertonatti, C. and J. Corcuera. 2000. Situación Ambiental Argentina 2000. Buenos Aires. Fundación Vida Silvestre Argentina. 439 pp.

Cabrera, A. L. 1976. Regiones Fitogeográficas Argentinas. Enciclopedia Argentina de Agricultura y Jardinería. Buenos Aires. Editorial ACME. T. II. 85 pp.

Cabrera, M. R. 1995. Comparative composition of turtle species in four natural regions of the Chacoan domain, South America. Anales del Museo de Historia Natural de Valparaiso 23: 41-52.

Cei, J. M. 1980. Amphibians of Argentina. Monitore Zoologico Italiano Monografia 2: 1-609.

Cei, J. M. 1993. Reptiles del noroeste, nordeste y este de Argentina. Herpetofauna de las Selvas Subtropicales, Puna y Pampas. Museo Regionale di Scienze Naturali Bollettino (Torino) 14: 1-949. 
Chebez, J. C., N. R. Rey and J. D. Williams. 2005. Reptiles de los Parques Nacionales de la Argentina. Buenos Aires. L.O.L.A. 76 pp.

Ferraro, D. P. and J. D. Williams. 2006. Material tipo de la Colección de Herpetología del Museo de La Plata, Buenos Aires, Argentina. Cuadernos de Herpetología 19 (2): 19-36.

Fitzgerald, L. A. 1994. Tupinambis lizards and people: a sustainable use approach to conservation and development. Conservation Biology 8: 12-15.

Gallardo, J. M. 1979. Composición, distribución y origen de la herpetofauna chaqueña. Pp. 299-307 in W. E. Duellman (ed.), The South American Herpetofauna Its origin, Evolution and Dispersal. Monographs of the Museum of Natural History of Kansas 7. 485 pp.

Giraudo, A. R. 2001. Serpientes de la Selva Paranaense y del Chaco Húmedo. Buenos Aires. L.O.L.A. 328 pp.

IUCN, Conservation International, and NatureServe. 2006 Global Amphibian Assessment. URL: www.globalam phibians.org. Captured on 04 May 2006.

Kretzschmar, S. 2006. Revisión y redescripción de Leptotyphlops albipunctus (Serpentes: Leptotyphlopidae). Cuadernos de Herpetología 19: 43-56.

Lavilla, E. O. and G. J. Scrocchi. 1991. Aportes a la herpetofauna del Chaco argentino: II- Nuevos datos sobre Odontophrynus lavillai Cei, 1985 (Anura: Leptodactylidae). Acta Zoologica Lilloana 40: 33-37.

Lavilla, E. O. and M. Rougés. 1992. Reproducción y Desarrollo de Anuros Argentinos. Asociación Herpetológica Argentina. Serie Divulgación 5. 66 pp.

Lavilla, E. O., F. B. Cruz and G. J. Scrocchi. 1995a. Amphibiens et reptiliens de la station biologique Los Colorados dans la province de Salta, Argentine. Revue Française d'Aquariologie et Herpetologie 22: 51-58.

Lavilla, E. O., F. B. Cruz and G. J. Scrocchi. 1995b. Amphibiens et reptiliens de la station biologique Los Colorados dans la province de Salta, Argentine $\left(2^{\mathrm{e}}\right.$ partie). Revue Française d'Aquariologie et Herpetologie 22: 117-128.

Lavilla, E. O., E. Richard and G. J. Scrocchi (eds.). 2000. Categorización de los Anfibios y Reptiles de la República Argentina. San Miguel de Tucumán. Asociación Herpetológica Argentina. 97 pp.

Leynaud, G. C. and E. H. Bucher. 1999. La Fauna de Serpientes del Chaco Sudamericano: diversidad, distribución geográfica y estado de conservación. Academia Nacional de Ciencias, Córdoba. Miscelánea 98. $46 \mathrm{pp}$.

Leynaud, G. C. and E. H. Bucher. 2005. Restoration of degraded Chaco woodlands: Effects on reptiles assemblages. Forest Ecology and Management 213: 384-390.

Morello, J. and J. Adámoli. 1974. Las Grandes Unidades de Vegetación y Ambientes del Chaco Argentino. Vegetación y ambiente de la Provincia del Chaco. INTA. Serie Fitogeográfica 13. 130pp.

Nenda, S. J. and G. J. Sccrochi. 2004. Nueva localidad para Leptodeira annulata pulchriceps en Argentina. Cuadernos de Herpetología 18: 77.

Pennington R. T., Darién E. P. and C. A. Pendry. 2000. Neotropical seasonally dry forests and Quaternary vegetation changes. Journal of Biogeography 27: 261274.

Perotti, M. G. 1997. Modos reproductivos y variables reproductivas cuantitativas de un ensamble de anuros del Chaco semiárido, Salta, Argentina. Revista Chilena de Historia Natural 70: 277-288.

Richard, E. 1999. Tortugas de las Regiones Áridas de Argentina: contribución al conocimiento de las tortugas de las regiones áridas de Argentina (Chelidae y Testudinidae) con especial referencia a los aspectos etoecológicos, comerciales y antropológicos de las especies del complejo chilensis (Chelonoidis chilensis y $\mathrm{C}$. donosobarrosi) en la provincia de Mendoza. Buenos Aires. L.O.L.A. 224 pp.

SADS (Secretaría de Ambiente y Desarrollo Sustentable). 2005. Resolución 1030/2004. Boletín Oficial, 11 de Enero $20051^{\circ}$ sección: 4.

SGPC (Secretaría Gobernación de la Provincia del Chaco). 2001. Boletín oficial. URL: http://www.chaco.gov.ar/ boletinoficial/Anio/2001/06/15-06-01.pdf. Captured on 13 June 2006.

SGPC (Secretaría Gobernación de la Provincia del Chaco). 2004. Boletín oficial. URL: http://www.chaco.gov.ar/ BoletinOficial/Anio/2004/12/13-12-04-8268.pdf. Captured on 13 June 2006.

Zak, M. R. and M. Cabido. 2002. Spatial patterns of the Chaco vegetation of central Argentina: Integration of remote sensing and phytosociology. Applied Vegetation Science 5: 213-226. 


\section{Appendix I - Material Examined}

Material examined is presented with collection number and locality. All specimens are deposited at Museo de La Plata (MLP). MLP codes: cf (photographic collection); A (amphibians); and R, S and JW (reptiles). For more information about the MLP herpetological codes, see Ferraro and Williams (2006).

\section{AMPHIBIANS}

Chaunus granulosus major MLP. (cf) 0001 (Loro Hablador Provincial Park); Chaunus schneideri MLP. (cf) 0005 (Loro Hablador Provincial Park); Trachycephalus venulosus MLP. A 1534 (Fuerte Esperanza), (cf) 0002 (Fuerte Esperanza); Phyllomedusa hypochondrialis azurea MLP. A 1544 (Taco Pozo), (cf). 0003 (Taco Pozo); Phyllomedusa sauvagii MLP. A 1537 (Taco Pozo), (cf) 0004 (Taco Pozo); Scinax nasicus MLP. (cf) 0006 (Taco Pozo); Ceratophrys cranwelli MLP-A-1525 (Taco Pozo), (cf) 0007 (Taco Pozo); Lepidobatrachus llanensis MLP. A 1523 (Taco Pozo), A-1528 (Taco Pozo); Leptodactylus bufonius MLP. (cf) 0009 (Loro Hablador Provincial Park); Leptodactylus chaquensis MLP. A 1526 (Taco Pozo), (cf) 0010 (Taco Pozo); Leptodactylus fuscus MLP. A 1531 (Taco Pozo), A 3454 (Fuerte Esperanza), (cf) 0011 (Fuerte Esperanza); Leptodactylus latinasus MLP. A 3460 (Fuerte Esperanza); Leptodactylus mystacinus MLP. A 3453 (Fuerte Esperanza), (cf) 0013 (Fuerte Esperanza); Odontophrynus lavillai MLP. (cf) 0012 (Loro Hablador Provincial Park); Physalaemus cuqui MLP. A 3455 (Puesto Primavera); Physalaemus biligonigerus MLP. A 3456 (Fuerte Esperanza), A 3457 (Fuerte Esperanza), A 3458 (Fuerte Esperanza), A 3459 (Fuerte Esperanza); Dermatonotus muelleri MLP. (cf) 0014 (Loro Hablador Provincial Park); Elachistocleis aff. bicolor MLP. (cf)-0003 (Taco Pozo); Pseudis paradoxus occidentalis MLP. (cf) 0008 (Río Muerto).

\section{REPTILES}

Chelonoidis chilensis MLP.(cf) 0019 (Loro Hablador Provincial Park); Polychrus acutirostris MLP.S 2223 (Loro Hablador Provincial Park); Liolaemus chacoensis MLP.R 5183 (Loro Hablador Provincial Park), (cf) 0022 (Loro Hablador Provincial Park); Tropidurus etheridgei MLP.S 2549 (Loro Hablador Provincial Park), ; Tropidurus spinulosus; MLP.R 5094 (Loro Hablador Provincial Park), S 2221 (Loro Hablador Provincial Park), S 2222 (Loro Hablador Provincial Park); (cf) 0024 (Loro Hablador Provincial Park); Stenocercus doellojuradoi MLP.R 5185 (Loro Hablador Provincial Park); Ameiva ameiva MLP.R 5206 (Loro Hablador Provincial Park); Cnemidophorus ocellifer MLP R 5190 (Loro Hablador Provincial Park); Teius teyou MLP.S 2547 (Loro Hablador Provincial Park), S 2550 (Loro Hablador Provincial Park), S 2224 (Loro Hablador Provincial Park), R 5154 (Loro Hablador Provincial Park), R 5155 (Loro Hablador Provincial Park), R 5205 (Loro Hablador Provincial Park), (cf) 0025 (Loro Hablador Provincial Park); Tupinambis rufescens MLP.(cf) 0026 (Loro Hablador Provincial Park), Vanzosaura rubricauda MLP.S 2548 (Loro Hablador Provincial Park), R 5180 (Loro Hablador Provincial Park); Mabuya frenata MLP.R 5204 (Loro Hablador Provincial Park), (cf) 0027 (Loro Hablador Provincial Park); Homonota fasciata MLP.R 5184 (Loro Hablador Provincial Park), (cf) 0028 (Loro Hablador Provincial Park); Phyllopezus pollicaris przewaIskyi MLP.R 5093 (Loro Hablador Provincial Park), R 5181 (Loro Hablador Provincial Park), R 5182 (Loro Hablador Provincial Park), R 5186 (Loro Hablador Provincial Park); Amphisbaena bolivica MLP.R 5156 (Loro Hablador Provincial Park); Anops kingi MLP.S 2225 (Fuerte esperanza); Leptotyphlops unguirostris MLP.R 5188 (Loro Hablador Provincial Park); 
Leptotyphlops albipunctus MLP.R 5187 (Loro Hablador Provincial Park); Boa constrictor occidentalis MLP.(cf) 0030 (Loro Hablador Provincial Park); Epicrates cenchria alvarezi MLP.R 5153 (Loro Hablador Provincial Park), (cf) 0031 (Loro Hablador Provincial Park); Boiruna maculata MLP.R 5203 (Loro Hablador Provincial Park), (cf) 0032 (Loro Hablador Provincial Park); Chironius quadricarinatus maculoventris MLP.(cf) 0033 (Loro Hablador Provincial Park); Leptodeira annulata pulchriceps MLP.JW 1913 (Loro Hablador Provincial Park); Liophis poecilogyrus caesius MLP.R 5177 (Loro Hablador Provincial Park); Liophis sagittifer modestus MLP.(cf) 0045 (Loro Hablador Provincial Park); Lystrophis pulcher MLP.R 5095 (Loro Hablador Provincial Park), (cf) 0034 (Loro Hablador Provincial Park); Oxyrhopus rhombifer MLP. JW 2001 (Loro Hablador Provincial Park); Philodryas baroni MLP.R 5152 (Loro Hablador Provincial Park), R 5175 (Loro Hablador Provincial Park),
, JW 1948 (Loro Hablador Provincial Park), (cf) 0035 (Loro Hablador Provincial Park); Philodryas mattogrossensis MLP.R 5189 (Loro Hablador Provincial Park), (cf) 0036 (Loro Hablador Provincial Park); Philodryas olfersii latirostris MLP.JW 1912 (Loro Hablador Provincial Park); Philodryas psammophidea MLP.R 5176 (Loro Hablador Provincial Park); Phimophis vittatus MLP.JW 2000 (Loro Hablador Provincial Park), (cf) 0038 (Loro Hablador Provincial Park); Sibynomorphus lavillai MLP.(cf) 0046 (Loro Hablador Provincial Park); Waglerophis merremii MLP. JW 2002 (Loro Hablador Provincial Park), (cf) 0039 (Loro Hablador Provincial Park); Micrurus pyrrhocryptus MLP. JW 1914 (Loro Hablador Provincial Park), (cf) 0040 (Loro Hablador Provincial Park); Bothrops neuwiedii diporus MLP. R 5151 (Loro Hablador Provincial Park), (cf) 0041 (Loro Hablador Provincial Park); Crotalus durissus terrificus MLP. (cf) 0042 (Loro Hablador Provincial Park). 